TRANSACTIONS OF THE

AMERICAN MATHEMATICAL SOCIETY

Volume 359, Number 4, April 2007, Pages 1829-1849

S 0002-9947(06)03950-X

Article electronically published on November 22, 2006

\title{
COMPLETENESS OF SYSTEMS OF COMPLEX EXPONENTIALS AND THE LAMBERT $W$ FUNCTIONS
}

\author{
ANDRÉ BOIVIN AND HUALIANG ZHONG
}

\begin{abstract}
We study some of the properties of the solution system $\left\{e^{i \lambda_{n} t}\right\}$ of the delay-differential equation $y^{\prime}(t)=a y(t-1)$. We first establish some general results on the stability of the completeness of exponential systems in $L^{2}$ and then show that the solution system above is always complete, but is not an unconditional basis in $L^{2}(-1 / 2,1 / 2)$.
\end{abstract}

\section{INTRODUCTION}

It is well known that the solutions of differential-difference equations can be expressed as (an infinite) sum of exponentials $\sum c_{n} e^{i \lambda_{n} t}$ where each $i \lambda_{n}$ is a characteristic root of the equation. For a "nice" initial condition $g(t)$, say, belonging to $C^{0}$, or $C^{1}$, the summation is known to converge pointwise to the solution it represents (see, for example, Bellman and Cooke [2]). In an attempt to better understand this representation, we were led to study the structure (e.g. completeness, basis or frame properties) of the exponential systems $\left\{e^{i \lambda_{n} t}\right\}$ when the $i \lambda_{n}$ have a distribution similar to that of the characteristic roots of $y^{\prime}(t)=a y(t-1)$.

The study of exponential systems, often referred to as the theory of nonharmonic Fourier series (see 23, 26]), has its origin in the classical works of R. Paley and $\mathrm{N}$. Wiener [15] and N. Levinson 13. One of the famous early results in the theory is that the basis property of the trigonometric system $\left\{e^{i n t}\right\}_{-\infty}^{\infty}$ is stable in $L^{2}(-\pi, \pi)$ in the sense that the system $\left\{e^{i \lambda_{n} t}\right\}_{-\infty}^{\infty}$ will always form a Riesz basis for $L^{2}(-\pi, \pi)$ if $\left|\lambda_{n}-n\right| \leq L<\frac{1}{4}$. M.I. Kadec' [9, and R.M. Redheffer and R.M. Young [17] have shown $\frac{1}{4}$ to be optimal. Many (but not all) of the subsequent results on the completeness, frames, basis or interpolation properties of exponential systems required that the $\lambda_{n}$ 's be located in a strip parallel to the real axis (e.g. 26]) or nearby the zeroes of a function of sine type (e.g. [1]). These results did not apply to our case since for the sequences we wish to consider, the $\lambda_{n}$ 's are located in a curvilinear strip. The few known results that allowed for a (slow) growth of the imaginary part of the $\lambda_{n}$ did not apply directly to our setting either and first needed to be extended. One of the main purposes of this paper is to provide such an extension.

The paper is organized as follows. Section 2 contains basic definitions and notations, and a discussion of the characteristic roots of the equation $y^{\prime}(t)=a y(t-1)$

Received by the editors July 4, 2003 and, in revised form, February 4, 2005.

2000 Mathematics Subject Classification. Primary 42C15, 42C30, 34K07; Secondary 30B50.

The first author was partially supported by a grant from NSERC of Canada.

(C)2006 American Mathematical Society Reverts to public domain 28 years from publication 
in terms of the Lambert $W$ functions, including a result on the asymptotic distributions of the roots. In Sections 3 and 4, we present and prove two theorems regarding the stability of the completeness property in $L^{2}$ of exponential systems under perturbations within a curvilinear strip. Previously imposed conditions in theorems of A.M. Sedletskii [18, and N. Fujii, A. Nakamura and R. Redheffer [6] and A.M. Sedletskii [23, §5.4, Theorem 5] have been relaxed. In Section 5, we apply Theorem 3.2 to special sequences related to the Lambert $W$ functions $W_{n}(a)$ introduced in Section 2. In particular, we show the completeness in $L^{2}\left(-\frac{1}{2}, \frac{1}{2}\right)$ of the system $\left\{e^{W_{n}(a) t}\right\}$. In Section 6, we show that the radius of completeness of the sequence $\left\{-i W_{n}(a)\right\}$ is equal to $\frac{1}{2}$, and thus that the main results of Section 5 cannot be derived from the powerful theorem of Beurling and Malliavin [3, 10.

\section{Preliminaries}

In this paper, we denote by $\mathbf{C}$ (respectively by $\mathbf{R}$ ) the set of all complex (respectively real) numbers. $\mathbf{Z}$ denotes the set of all integers, and $\sum^{\prime}$ and $\Pi^{\prime}$ mean summation and multiplication, respectively, through all the integers except 0 . Unless otherwise specified, all sequences considered in this paper will be indexed by the integers from $-\infty$ to $\infty$.

We say that an entire function $f(z)$ is of exponential type $\gamma$ if there is a constant $A>0$ such that

$$
|f(z)| \leq A e^{\gamma|z|}
$$

The totality of all entire functions of exponential type at most $\pi$ that are square integrable on the real axis is known as the Paley-Wiener space (see [26) which is a Hilbert space with respect to the inner product $(f, g)=\int_{-\infty}^{\infty} f(x) \overline{g(x)} d x$.

A system $\left\{e^{i \lambda_{n} t}\right\}$ of complex exponentials is closed in $L^{p}(-\gamma, \gamma), 1 \leq p<\infty$, if every $f \in L^{p}(-\gamma, \gamma)$ can be approximated in $L^{p}$ norm by (finite) linear combinations of the functions $e^{i \lambda_{n} t}$.

A system $\left\{e^{i \lambda_{n} t}\right\}$ of complex exponentials is complete in $L^{p}(-\gamma, \gamma), 1 \leq p<\infty$, if the relations

$$
\int_{-\gamma}^{\gamma} f(t) e^{i \lambda_{n} t} d t=0
$$

for all $n$ with $f \in L^{p}$ imply that $f=0$ a.e. In this case $\left\{\lambda_{n}\right\}$ is called a complete sequence.

Duality shows that closure in $L^{q}$ is equivalent to completeness in $L^{p}$ if $\frac{1}{p}+\frac{1}{q}=1$, and $1<p<\infty$. Especially, when $p=q=2$, completeness is equivalent to closure in $L^{2}(-\gamma, \gamma)$ (see [13] or [26]).

The following important result of N. Levinson establishes the deep connection that exists between completeness of sets of exponentials and entire functions.

Proposition A (N. Levinson, [13]). For the system $\left\{e^{i \lambda_{n} t}\right\}$ to be incomplete in $C(-\gamma, \gamma)$ (or in $\left.L^{p}(-\gamma, \gamma), 1<p<\infty\right)$, it is necessary and sufficient that there exists a non-trivial entire function $f(z)$, which vanishes at every $\lambda_{n}$ and is expressible 
in the form

$$
f(z)=\int_{-\gamma}^{\gamma} e^{i z t} d \omega(t)
$$

where $\omega(t)$ is of bounded variation on $(-\gamma, \gamma)$ (or $\left.\omega(t) \in L^{q}(-\gamma, \gamma), 1 / p+1 / q=1\right)$.

Note 2.1. Equation (2.1) defines a function of exponential type at most $\gamma$ and thus, when $p=2$ and $\gamma=\pi$, the Plancherel Theorem shows that $f$ in fact belongs to the Paley-Wiener space.

Definition 2.2. A complete system $\left\{e^{i \lambda_{n} t}\right\}$ in $L^{p}$ that ceases to be complete when any one of its element is removed is said to be an exact complete system. When exactly $m$ elements have to be removed (or added) in order that the new system be exact, then the excess $E_{p}(\lambda)$ of the system is $m$ (or $-m$ ).

Definition 2.3. A sequence $\left\{f_{1}, f_{2}, \ldots\right\}$ in an infinite-dimensional Banach space $X$ is said to be a Schauder basis for $X$ if for each $f \in X$, there is a unique sequence of scalars $\left\{c_{1}, c_{2}, \ldots\right\}$ such that $f=\sum_{n=1}^{\infty} c_{n} f_{n}$, i.e.

$$
\left\|f-\sum_{i=1}^{n} c_{i} f_{i}\right\| \rightarrow 0 \quad \text { as } \quad n \rightarrow \infty
$$

Henceforth, the term basis will always mean a Schauder basis.

To study the completeness of the solution system of the delay-differential equation $y^{\prime}(t)=a y(t-1)$, where $a$ is a nonzero fixed real number, we need to understand the zeroes of its characteristic function $g(z)=z-a e^{-z}$ as a function of the variable $a$ as well as their asymptotic distribution.

Note that when $a \neq-\frac{1}{e}$, all the zeroes of $g(z)$ are simple and that when $a=-\frac{1}{e}$, there is a double zero at $z=-1$. Assuming first that $a \neq-\frac{1}{e}$ and that $g(z)=0$, and writing $z$ as $z=\eta_{1}+i \eta_{2}=r e^{i \phi}(-\pi<\phi \leq \pi)$, we get that

$$
\eta_{1}=\log |a|-\log r \quad \text { and } \quad \eta_{2}=\arg a+2 \pi n-\phi
$$

for some $n \in \mathbf{Z}$. Now assume that $a$ is real. It is known (see 25] or [24]) that:

(1) If $a$ does not satisfy $-\frac{1}{e} \leq a \leq 0$, then there is a zero $z_{n}$ corresponding to each integer $n \in \mathbf{Z}$.

(2) If $a$ satisfies $-\frac{1}{e}<a<0$, then there is a zero $z_{n}$ corresponding to each integer $n$ in $\mathbf{Z} \backslash\{-1,0\}$, and to $n=0$ there correspond two zeroes $z_{0}$ and $z_{-1}$ satisfying $-1<z_{0}<0$ and $z_{-1}<-1$. Noting that from (2.2) we cannot have $n=-1$ when $a<0$ and $z<0$, we still have a one-to-one correspondence between the zeroes of $g$ and all the integers.

(3) If $a=-\frac{1}{e}$, there is a zero of order two at -1 , and thus after setting $z_{0}=z_{-1}=-1$, the zeroes of $g$ are again in exact correspondence with all the integers.

To each integer $n$, there thus corresponds a unique zero of $g$ which depends on the variable $a$. This defines a function $W_{n}(a)$ called the Lambert $W$ function (see [5]). The following asymptotic properties of these functions were obtained by S. Verblunsky in 1961. 
Proposition B (S. Verblunsky, 24]). Suppose $W_{n}(a)$ is the Lambert $W$ function for $n \in \mathbf{Z}$, and $\alpha=\frac{|a|}{2 \pi}$. Then

i) when $a>0$, for all $n \neq 0$, we have

$$
\begin{aligned}
W_{n}(a)= & \left\{\log \frac{\alpha}{|n|}+\frac{1}{4 n} \operatorname{sign}(n)+O\left(\frac{\log |n|}{n}\right)^{2}\right\} \\
& +i\left\{2 \pi\left(n-\frac{1}{4} \operatorname{sign}(n)\right)+\frac{\log \frac{\alpha}{|n|}}{2 \pi n}+O\left(\frac{\log |n|}{n^{2}}\right)\right\},
\end{aligned}
$$

and $W_{0}(a)>0$

ii) when $-\frac{1}{e}<a<0$, for all $n \in \mathbf{Z} \backslash\{-1,0\}$, we have

$$
\begin{aligned}
W_{n}(a)= & \left\{\log \frac{\alpha}{|n|}-\left(\frac{1}{2 n}-\frac{1}{4 n} \operatorname{sign}(n)\right)+O\left(\frac{\log |n|}{n}\right)^{2}\right\} \\
& +i\left\{2 \pi\left(n+\frac{1}{2}-\frac{1}{4} \operatorname{sign}(n)\right)+\frac{\log \frac{\alpha}{|n|}}{2 \pi n}+O\left(\frac{\log |n|}{n^{2}}\right)\right\},
\end{aligned}
$$

and $-1<W_{0}(a)<0, W_{-1}(a)<-1$;

iii) when $a=-\frac{1}{e}$, we have (2.3) for all $n \in \mathbf{Z} \backslash\{-1,0\}$, and $W_{0}(a)=W_{-1}(a)=$ $-1$

iv) when $a<-\frac{1}{e}$, we have (2.3) for all $n \in \mathbf{Z} \backslash\{0\}$, and $W_{0}(a)$ is not real.

Now for a nonzero real number $a$, we know that if $z_{n}$ satisfies $z_{n} e^{z_{n}}=a$, then $\overline{z_{n}}$ also satisfies $\overline{z_{n}} e^{\overline{z_{n}}}=a$. It follows that

(1) if $a>0, W_{0}(a)$ is real, and $\overline{W_{n}(a)}=W_{-n}(a)$ for all $n \geq 1$;

(2) if $-\frac{1}{e} \leq a<0$, only $W_{0}(a)$ and $W_{-1}(a)$ are real, and for all $n \geq 1$, we have $\overline{W_{n}(a)}=W_{-n-1}(a)$;

(3) if $a<-\frac{1}{e}$, none of the $W_{n}(a)$ are real, and we have that $\overline{W_{n}(a)}=W_{-n-1}(a)$ for all $n \geq 0$.

For convenience, we modify the sequence and rescale the $W_{n}(a)$ in the following ways: When $a>0$, we set

$$
V_{n}(a)=-\frac{i}{2 \pi} W_{n}(a) \quad \text { for } \quad n \in \mathbf{Z} .
$$

When $a<0$, we set

$$
V_{n}(a)=\left\{\begin{array}{cc}
-\frac{i}{2 \pi} W_{n}(a), & n>0, \\
0, & n=0, \\
-\frac{i}{2 \pi} W_{n-1}(a), & n<0 .
\end{array}\right.
$$

Furthermore we set $\rho_{n}(a)=\operatorname{Re} V_{n}(a)$ and $\sigma_{n}(a)=\operatorname{Im} V_{n}(a)$.

It follows that when $a<0,\left\{V_{n}(a)\right\}$ has "one element less" than $\left\{W_{n}(a)\right\}$. But it gives us a nice symmetrical index on both sequences $\left\{\rho_{n}(a)\right\}$ and $\left\{V_{n}(a)\right\}$. Indeed, from the definition of $V_{n}(a)$ and Proposition B, we immediately obtain:

Lemma 2.4. Define $V_{n}(a)$ and $\rho_{n}$ as above. Then $\overline{V_{n}(a)}=-V_{-n}(a)$ and $\rho_{n}(a)=$ $-\rho_{-n}(a)$ for all integers $n$ and (nonzero) real a. Moreover $V_{n}(a)$ has the following asymptotic property. 
When $a>0$,

$$
V_{n}(a)= \begin{cases}\left\{n-\frac{1}{4}-\varepsilon_{n}^{(1)}(a)\right\}+i\left\{\frac{1}{2 \pi} \log |n|+O(1)\right\}, & n>0, \\ \left\{n+\frac{1}{4}+\varepsilon_{n}^{(1)}(a)\right\}+i\left\{\frac{1}{2 \pi} \log |n|+O(1)\right\}, & n<0 .\end{cases}
$$

When $a<0$,

$$
V_{n}(a)=\left\{\begin{array}{lc}
\left\{n+\frac{1}{4}-\varepsilon_{n}^{(2)}(a)\right\}+i\left\{\frac{1}{2 \pi} \log |n|+O(1)\right\}, & n>0, \\
\left\{n-\frac{1}{4}+\varepsilon_{n}^{(2)}(a)\right\}+i\left\{\frac{1}{2 \pi} \log |n|+O(1)\right\}, & n<0 .
\end{array}\right.
$$

Here $\varepsilon_{n}^{(j)}(a)=O\left(\frac{\log |n|}{|n|}\right)$ and $\varepsilon_{n}^{(j)}(a)>0, j=1,2$, for sufficiently large $n$ depending on $a$.

We end this section by noting that the above rescaling of $W_{n}(a)$ is of no consequence for the theory that follows. Indeed we have:

\section{Lemma 2.5.}

(1) Let $\mu_{n}=\sigma \lambda_{n}, \sigma>0$. If $\left\{e^{i \lambda_{n} t}\right\}$ is complete in $L^{p}(-\gamma, \gamma), 1<p<\infty$, then $\left\{e^{i \mu_{n} t}\right\}$ is complete in $L^{p}(-\gamma / \sigma, \gamma / \sigma), 1<p<\infty$.

(2) Let $\mu_{n}=\lambda_{n}+\tau$. If $\left\{e^{i \lambda_{n} t}\right\}$ is complete in $L^{p}(-\gamma, \gamma), 1<p<\infty$, then $\left\{e^{i \mu_{n} t}\right\}$ is complete in $L^{p}(-\gamma-\tau, \gamma-\tau), 1<p<\infty$.

Proof. Let $\mu_{n}=\sigma \lambda_{n}$. If $\left\{e^{i \mu_{n} t}\right\}$ is not complete in $L^{p}\left(-\frac{\gamma}{\sigma}, \frac{\gamma}{\sigma}\right)$, then by Proposition A, there exists an entire function $g$ of the form

$$
g(z)=\int_{-\frac{\gamma}{\sigma}}^{\frac{\gamma}{\sigma}} e^{i z t} d \omega(t)
$$

such that $g\left(\mu_{n}\right)=0$ where $\omega(t)$ is in $L^{q}\left(-\frac{\gamma}{\sigma}, \frac{\gamma}{\sigma}\right)$ and $1 / p+1 / q=1$.

Substituting $\sigma w$ for $z$ in the above integral, we obtain

$$
g_{1}(w)=g(\sigma w)=\int_{-\frac{\gamma}{\sigma}}^{\frac{\gamma}{\sigma}} e^{i \sigma w t} d \omega(t)=\int_{-\gamma}^{\gamma} e^{i w s} d \omega_{1}(s),
$$

where $\omega_{1}(s)=\omega(s / \sigma)$ is in $L^{q}(-\gamma, \gamma)$, and $s=\sigma t$. So $g_{1}$ is an entire function satisfying $g_{1}\left(\lambda_{n}\right)=g\left(\mu_{n}\right)=0$. By Proposition A, $\left\{e^{i \lambda_{n} t}\right\}$ is incomplete in $L^{p}(-\gamma, \gamma)$, which is a contradiction.

The proof of (2) is the same as that for (1) provided this time we substitute $z+\tau$ for $z$ in the integral.

\section{Stability of COMPleteness - PART I}

N. Levinson (see [13]) showed that $\left\{e^{i \lambda_{n} t}\right\}$ is complete in $L^{2}(-\pi, \pi)$ if $\left|\lambda_{n}-n\right| \leq$ $\frac{1}{4}$, and incomplete if $\lambda_{n}=n+\left(\frac{1}{4}+\epsilon\right) \operatorname{sign}(n)$ for $n \neq 0$ and arbitrary constant $\epsilon>0$, and $\lambda_{0}=0$. So it is not too surprising that the two systems $\left\{e^{i \lambda_{n}^{+} t}\right\}$ and $\left\{e^{i \lambda_{n}^{-} t}\right\}$ have received a lot of attention, where the $\lambda_{n}^{+}$and $\lambda_{n}^{-}$are defined by

$$
\lambda_{n}^{+}= \begin{cases}n+\operatorname{sign}(n) \frac{1}{4}, & n \in \mathbf{Z} /\{0\}, \\ 0, & n=0,\end{cases}
$$


and

$$
\lambda_{n}^{-}= \begin{cases}n-\operatorname{sign}(n) \frac{1}{4}, & n \in \mathbf{Z} /\{0\}, \\ 0, & n=0 .\end{cases}
$$

See for example [17, 18, 20, 22, 26, 27, 28].

In 1940, N. Levinson gave the following sufficient condition for completeness.

Proposition C (N. Levinson, [13]). Suppose that $\left\{\lambda_{n}\right\}$ is a sequence of complex numbers, that $n_{\lambda}(t)$ denotes the number of points $\lambda_{k}$ in the disc $|z| \leq t$ and let $N(R)=\int_{0}^{R} \frac{n_{\lambda}(t)}{t} d t$. If the sequence $\left\{\lambda_{n}\right\}$ satisfies

$$
\limsup _{R \rightarrow \infty}\left\{N(R)-\frac{2 \gamma}{\pi} R+\frac{1}{p} \ln R\right\}>-\infty,
$$

then the system $\left\{e^{i \lambda_{n} t}\right\}$ is complete in $L^{p}(-\gamma, \gamma)(1<p<\infty)$. Especially if $\lambda_{n}$ satisfy $\left|\lambda_{n}\right| \leq|n|+\frac{1}{2 p}, n \in \mathbf{Z}$, then the system $\left\{e^{i \lambda_{n} t}\right\}$ is complete in $L^{p}(-\pi, \pi)$. The constant $\frac{1}{2 p}$ is the best possible.

Remark 3.1. It follows from Proposition $\mathrm{C}$ that if the sequence $\left\{\lambda_{n}\right\}$ is either $\left\{\lambda_{n}^{+}\right\}$or $\left\{\lambda_{n}^{-}\right\}$as defined by (3.1) and (3.2), then the system $\left\{e^{i \lambda_{n} t}\right\}$ is complete in $L^{2}(-\pi, \pi)$.

We note also that although $1 /(2 p)$ is the best possible constant, the condition above can still be relaxed if the constant is replaced by a sequence depending on $n$.

Theorem A (A.M. Sedletskii, [18). Let $\left\{\lambda_{n}\right\}$ and $\left\{\mu_{n}\right\}$ be two real sequences and $0<\gamma<\infty$. Then the excess $E_{2}(\lambda)$ is equal to the excess $E_{2}(\mu)$ in $L^{2}(-\gamma, \gamma)$ if either one of the following conditions holds:

(1) For some $0<s<\infty, \sum\left|\lambda_{n}-\mu_{n}\right|^{s}<\infty$.

(2) $\left|\lambda_{n}-\mu_{n}\right| \leq \alpha_{n}, \alpha_{n} \rightarrow 0$, as $|n| \rightarrow \infty$ and $\sum^{\prime} \frac{\alpha_{n}}{|n|}<\infty$.

Now we wish to extend this last case to complex sequences. This will be needed in our proof of Theorem 5.1 .

Theorem 3.2. Let $0<\gamma<\infty$, and let $\left\{\lambda_{n}\right\}$ and $\left\{\mu_{n}\right\}$ be two sequences of distinct complex numbers such that

$$
\left|\lambda_{n}-\mu_{n}\right| \leq \phi(|n|)
$$

where $\phi(|n|)$ is nonincreasing and tends to 0 , and $\sum^{\prime} \frac{\phi(|n|)}{|n|}<\infty$. Then the excesses of the two exponential systems $\left\{e^{i \lambda_{n} t}\right\}$ and $\left\{e^{i \mu_{n} t}\right\}$ in $L^{2}(-\gamma, \gamma)$ are equal, that is, $E_{2}(\lambda)=E_{2}(\mu)$.

For the proof, we will need the following two results.

Proposition D (N. Levinson, [12, Proof of Theorem III], [13, Theorem VIII]). Suppose $f(z)$ is an entire function of exponential type $a$ and $\left\{\lambda_{n}\right\}$ is its zero set. Let $n^{+}(r)$ and $n^{-}(r)$ be the counting functions for its zeroes in the right half disc $\{z \in \mathbf{C}:|z|<r, \operatorname{Re} z \geq 0\}$ and in the left half disc $\{z \in \mathbf{C}:|z|<r, \operatorname{Re} z<0\}$, respectively. Let $n_{1}(r)$ and $n_{2}(r)$ be the numbers of points of the sequence $\left\{\frac{\left|\lambda_{n}\right|^{2}}{\rho_{n}}\right.$ : $\left.\rho_{n} \neq 0\right\}$ in $(-r, 0)$ and $(0, r)$, respectively, where $\rho_{n}=\operatorname{Re} \lambda_{n}$. If

$$
\lim _{\substack{|x| \rightarrow \infty \\ x \in \mathbf{R}}} \frac{\log |f(x)|}{|x|} \leq 0 \text { and } \int_{-\infty}^{\infty} \frac{\log ^{+}|f(x)|}{1+x^{2}} d x<\infty,
$$


then for some constant $C_{0}$, the following statements hold:

(1) $\lim _{r \rightarrow \infty} \frac{n^{+}(r)}{r}=\lim _{r \rightarrow \infty} \frac{n^{-}(r)}{r}=C_{0}$.

(2) $\lim _{r \rightarrow \infty} \frac{n_{1}(r)}{r}=\lim _{r \rightarrow \infty} \frac{n_{2}(r)}{r}=C_{0}$.

Remark 3.3. The function $f$ defined by (2.1) in Proposition A is bounded on the real axis, and thus such an $f$ satisfies the hypotheses of Proposition D. But by Bernstein's theorem (see [4, Notes for Chapter 3]), such an $f$ must be of nonzero type. The proof of Proposition $\mathrm{D}$ then yields in this case that $C_{0}>0$.

Proposition E (A.M. Sedletskii, 21]).

a) Suppose $G(z)$ is in the Paley-Wiener space and its zero set is $\left\{z_{n}\right\}_{n=0}^{\infty}$. Then $\lim _{r \rightarrow \infty} \sum_{0<\left|z_{n}\right|<r} \frac{1}{z_{n}}$ exists.

b) Furthermore, suppose the Hadamard's factorization of $G(z)$ is

$$
G(z)=B e^{a z}\left(z-z_{0}\right) \prod_{n=1}^{\infty}\left(1-\frac{z}{z_{n}}\right) .
$$

Assume the complex sequence $\left\{w_{n}\right\}$ satisfies $w_{0}=z_{0}, \lim _{n \rightarrow \infty} \sum_{\left|w_{n}\right|<n} \frac{1}{w_{n}}$ exists and $\lim _{n \rightarrow \infty} \frac{z_{n}}{w_{n}}=1$. Set

$$
P(z)=B e^{a z}\left(z-w_{0}\right) \prod_{n=1}^{\infty}\left(1-\frac{z}{w_{n}}\right) .
$$

If $|P(z)| \leq k|G(z)|$ on some horizontal line $\operatorname{Im} z=h$ with constants $k$ and $h$, then there is a real constant $\alpha$ such that the function $e^{i \alpha z} P(z)$ is in the Paley-Wiener space. Consequently, $\left\{e^{i w_{n} t}\right\}_{n=0}^{\infty}$ is incomplete in $L^{2}(-\pi, \pi)$.

Proof of Theorem 3.2 . To prove the theorem, we need only to show that $\left\{\mu_{n}\right\}$ is not a complete sequence in $L^{2}(-\gamma, \gamma)$ if $\left\{\lambda_{n}\right\}$ is assumed to be incomplete in $L^{2}(-\gamma, \gamma)$. Without loss of generality, we can assume $\gamma=\pi$.

So assume that $\left\{\lambda_{n}\right\}$ is incomplete in $L^{2}(-\pi, \pi)$. Then by Proposition A, there is an entire function $F(z)$ of the form (2.1) in the Paley-Wiener space such that the sequence $\left\{\lambda_{n}\right\}$ is a subset of its zeroes. Noting that for any zero point $\beta$ of $F(z), F(z) /(z-\beta)$ belongs to the Paley-Wiener space (see [13, [11, Appendix III, Theorem 2]), we can assume without loss of generality that $\left\{\lambda_{n}\right\}$ is exactly the zero set of $F$ and that all the zeroes are simple.

Since any finite number of terms can be replaced without altering the completeness (see [13]), considering Proposition D, we can assume that $\mu_{0}=\lambda_{0}=0$ and

$$
\operatorname{Re} \lambda_{n}<0 \text { for } n<0 \text { and } \operatorname{Re} \lambda_{n} \geq 0 \text { for } n>0 \text {. }
$$

We know from Proposition E that $\sum^{\prime} \frac{1}{\lambda_{n}}$ exists, and the Hadamard factorization of $F(z)$ gives that

$$
F(z)=B e^{a z} z \prod^{\prime}\left(1-\frac{z}{\lambda_{n}}\right) .
$$

Let $\lambda_{n}=\rho_{n}+i \sigma_{n}$. We first show that there exists a $\delta>0$ such that

$$
\frac{\delta}{2} \leq \frac{\left|\lambda_{n}\right|}{|n|} \leq 2 \delta
$$


and

$$
\frac{\delta}{8} \leq \frac{\left|\rho_{n}\right|}{|n|} \leq 8 \delta
$$

for $|n|$ large enough. Indeed, from Proposition D and Remark 3.3, we have that

$$
\frac{C_{0}}{2}<\frac{n^{+}\left(\left|\lambda_{n}\right|\right)}{\left|\lambda_{n}\right|}<2 C_{0} \quad \text { and } \quad \frac{C_{0}}{2}<\frac{n^{-}\left(\left|\lambda_{n}\right|\right)}{\left|\lambda_{n}\right|}<2 C_{0}
$$

for sufficiently large $n$. From our assumptions on the $\lambda_{n}$ 's, it follows that $n^{+}\left(\left|\lambda_{n}\right|\right)=$ $n^{-}\left(\left|\lambda_{-n}\right|\right) \approx|n|$. Thus, for large enough $n$,

$$
\frac{2}{C_{0}} \geq \frac{\left|\lambda_{n}\right|}{|n|} \geq \frac{1}{2 C_{0}}
$$

and (3.4) follows with $\delta=1 / C_{0}$.

Similarly, from (2) of Proposition D, we have $\frac{C_{0}}{2} \leq \frac{n \rho_{n}}{\left|\lambda_{n}\right|^{2}} \leq 2 C_{0}$. Combining with (3.4), we get (3.5).

Now from the hypotheses of the theorem and Proposition E, it follows that

$$
\lim _{n \rightarrow \pm \infty}\left|1-\frac{\mu_{n}}{\lambda_{n}}\right|=\lim _{n \rightarrow \pm \infty}\left|\frac{\lambda_{n}-\mu_{n}}{\lambda_{n}}\right| \leq \lim _{n \rightarrow \infty} \frac{2 \phi(|n|)}{\delta|n|}=0
$$

and

We now set

$$
\sum^{\prime} \frac{1}{\mu_{n}}=\sum^{\prime}\left(\frac{\lambda_{n}-\mu_{n}}{\lambda_{n} \mu_{n}}+\frac{1}{\lambda_{n}}\right) \leq \sum^{\prime} \frac{4 \phi(|n|)}{\delta^{2} n^{2}}+\sum^{\prime} \frac{1}{\lambda_{n}}<\infty .
$$

$$
F^{*}(z)=B e^{a z} z \prod^{\prime}\left(1-\frac{z}{\mu_{n}}\right) .
$$

To reach our goal we will apply the second part of Proposition E with $h=0$ and will show that $\left|F^{*}(x)\right| \leq$ const $|F(x)|, x \in \mathbf{R}$. By Proposition $\mathrm{F}$ below (see Section 5) we can assume $\left|\sigma_{n}\right| \geq 1$ for all $n$, and since by hypothesis the sequence $\{\phi(|n|)\}$ must be bounded, Proposition $\mathrm{F}$ again allows us to change the imaginary part of $\mu_{n}$ to agree with that of $\lambda_{n}$. So we may set $\mu_{n}=\lambda_{n}+\tau_{n}$ where $\tau_{n}$ is real. It follows that for $x \in \mathbf{R}$,

$$
\begin{aligned}
\left|1-\frac{x}{\mu_{n}}\right|^{2} & =\frac{\left(\rho_{n}-x+\tau_{n}\right)^{2}+\sigma_{n}^{2}}{\left(\rho_{n}-x\right)^{2}+\sigma_{n}^{2}}\left|\frac{\lambda_{n}}{\mu_{n}}\right|^{2}\left|1-\frac{x}{\lambda_{n}}\right|^{2} \\
& =\left(1+\eta_{n}\right)\left|1+\frac{\lambda_{n}-\mu_{n}}{\mu_{n}}\right|^{2}\left|1-\frac{x}{\lambda_{n}}\right|^{2}
\end{aligned}
$$

where $\eta_{n}=\left|2 \tau_{n}\left(\rho_{n}-x\right)+\tau_{n}^{2}\right| /\left\{\left(\rho_{n}-x\right)^{2}+\sigma_{n}^{2}\right\}$. Note that when $0 \leq u \leq b_{0}<1$, we have $\log (1+u)=\sum^{\prime} \frac{u^{k}}{k} \leq \frac{u}{1-b_{0}}$. So $\sum \log \left(1+u_{n}\right)$ is convergent if $\sum u_{n}$ is convergent.

Since $\frac{\left|\lambda_{n}\right|}{|n|} \geq \frac{\delta}{2}$ and $\left|\lambda_{n}-\mu_{n}\right| \leq \phi(|n|)$, the hypothesis on $\phi$ implies that $\sum \log \left|1+\frac{\lambda_{n}-\mu_{n}}{\mu_{n}}\right|$ converges. Next, since $\left|\eta_{n}\right| \leq \frac{\left|2 \tau_{n}\left(\rho_{n}-x\right)\right|}{\left(\rho_{n}-x\right)^{2}+\sigma_{n}^{2}}+\frac{\left|\tau_{n}^{2}\right|}{\left(\rho_{n}-x\right)^{2}+\sigma_{n}^{2}}$, to show that $\sum\left|\eta_{n}\right|$ is bounded on the positive real axis and that the bound is independent of $x$, we consider three cases:

1) When $\rho_{n}<\frac{x}{2},\left|x-\rho_{n}\right| \geq\left|\rho_{n}\right|=O(n)$. So from the hypothesis on $\phi$ and (3.5), we have that

$$
\sum_{\rho_{n}<\frac{x}{2}}\left|\eta_{n}\right| \leq \sum_{\rho_{n}<\frac{x}{2}} \frac{2 \phi(|n|)}{\left|\rho_{n}\right|}+\sum_{\rho_{n}<\frac{x}{2}} \frac{\phi^{2}(|n|)}{\left|\rho_{n}\right|^{2}}<C,
$$

which is uniformly bounded. 
2) When $\frac{x}{2} \leq \rho_{n}<x+1$, then

$$
\begin{aligned}
\sum_{\frac{x}{2} \leq \rho_{n}<x+1}\left|\eta_{n}\right| \leq & \left\{\sum_{\frac{x}{2} \leq \rho_{n}<x-1}+\sum_{x-1<\rho_{n}<x+1}\right\} \frac{2 \phi(|n|)\left|\rho_{n}-x\right|}{\left(x-\rho_{n}\right)^{2}+\left|\sigma_{n}\right|^{2}} \\
& +\left\{\sum_{\frac{x}{2} \leq \rho_{n}<x-1}+\sum_{x-1<\rho_{n}<x+1}\right\} \frac{\phi^{2}(|n|)}{\left(x-\rho_{n}\right)^{2}+\left|\sigma_{n}\right|^{2}} \\
\leq & \sum_{\frac{x}{2} \leq \rho_{n}<x-1} \frac{2 \phi(|n|)}{\left|\rho_{n}-x\right|}+\sum_{x-1<\rho_{n}<x+1} \frac{2 \phi(|n|)\left|\rho_{n}-x\right|}{\left|\rho_{n}-x\right|^{2}+1} \\
& +\sum_{\frac{x}{2} \leq \rho_{n}<x-1} \frac{\phi^{2}(|n|)}{\left|\rho_{n}-x\right|^{2}}+\sum_{x-1<\rho_{n}<x+1} \frac{\phi^{2}(|n|)}{\left|\rho_{n}-x\right|^{2}+1} \\
\leq & \int_{\frac{x}{2}}^{x-1} \frac{2 \phi\left(\frac{t}{8 \delta}\right)}{|t-x|} d n(t)+\int_{x-1}^{x+1} 2 \phi\left(\frac{t}{8 \delta}\right) d n(t) \\
& +\int_{\frac{x}{2}}^{x-1} \frac{\phi^{2}\left(\frac{t}{8 \delta}\right)}{|t-x|^{2}} d n(t)+\int_{x-1}^{x+1} \phi^{2}\left(\frac{t}{8 \delta}\right) d n(t) \\
= & I_{1}+I_{2}+I_{3}+I_{4},
\end{aligned}
$$

where $n(t)$ is the counting function of $\rho_{n}$ in the interval $(0, t)$.

Since $\phi(t)$ is nonincreasing, and $n(t) / t$ is bounded uniformly, as shown by (3.5), we have that both $I_{2}$ and $I_{4}$ are uniformly bounded. For $I_{1}$, again since $\phi(t)$ is nonincreasing, we have that

$$
I_{1} \leq \int_{\frac{x}{2}}^{x-1} \frac{2 \phi\left(\frac{x-t}{8 \delta}\right)}{x-t} d n(t) \leq \int_{1}^{\frac{x}{2}} \frac{2 \phi\left(\frac{s}{8 \delta}\right)}{s} d n(s) .
$$

Since $\sum^{\prime} \frac{\phi(|n|)}{|n|}<\infty$, we get that $I_{1}$ is uniformly bounded. Similarly, since $\phi(x)$ is nonincreasing, it follows that

$$
I_{3} \leq \phi\left(\frac{x}{16 \delta}\right) \int_{\frac{x}{2}}^{x-1} \frac{\phi\left(\frac{x-t}{8 \delta}\right)}{x-t} d n(t),
$$

which is uniformly bounded.

3) When $\rho_{n}>x+1$,

$$
\sum_{\rho_{n}>x+1}\left|\eta_{n}\right| \leq \sum_{\rho_{n}>x+1} \frac{\left|2 \phi(n)\left(\rho_{n}-x\right)\right|}{\left(\rho_{n}-x\right)^{2}+1}+\sum_{\rho_{n}>x+1} \frac{\left|\phi^{2}(n)\right|}{\left(\rho_{n}-x\right)^{2}+1} .
$$

Similar to the above discussion, we only need to establish a uniform bound for $I_{5}=\sum_{\rho_{n}>x+1} \frac{|\phi(n)|}{\left(\rho_{n}-x\right)}$. Since

$$
I_{5} \leq \int_{x+1}^{\infty} \frac{\phi\left(\frac{t}{8 \delta}\right)}{t-x} d n(t) \leq \int_{1}^{\infty} \frac{\phi\left(\frac{1}{8 \delta}(u+x)\right)}{u} d n(u)
$$

and since $\sum \frac{\phi(n)}{n}$ does converge and $\phi(x)$ is nonincreasing for $x>0$, we get that $I_{5} \leq \int_{1}^{\infty} \frac{\phi\left(\frac{1}{8 \delta}(u)\right)}{u} d n(u)$, which is uniformly bounded.

Thus we get $\left|F^{*}(x)\right| \leq$ const $|F(x)|$ for $x>0$. The same argument can be applied to the case of $x<0$. So $\left|F^{*}(x) / F(x)\right|$ is uniformly bounded on the real axis, and this completes the proof. 


\section{Stability of COMPleteness - Part II}

In the last section, we have discussed and compared the excesses of two complex sequences which were close to each other. In this section, we consider some cases where the corresponding elements of the two sequences are some fixed distance apart.

In 1999, N. Fujii, A. Nakamura and R. Redheffer published the following result.

Theorem B ([6]). Let $\left\{\mu_{n}\right\}$ be a complex sequence such that $\left|\mu_{n}-n\right| \leq c$ for $-\infty<n<\infty$. Let $\lambda_{0}=\mu_{0}$ and

$$
\lambda_{n}=\mu_{n}+a \quad \text { and } \quad \lambda_{-n}=\mu_{-n}-b \quad \text { for } \quad n>0,
$$

where $a \geq 0$ and $b \geq 0$ are constants. Then $E_{2}(\lambda) \leq E_{2}(\mu)$ on the interval $(-\pi, \pi)$.

This theorem is in fact a special case of a more general result obtained previously by A.M. Sedletskii $([20,23, \S 5.4$, Theorem 5]). We will extend the condition $\left|\mu_{n}-n\right| \leq c$ to $\left|\operatorname{Re} \mu_{n}-n\right| \leq c$, and prove the following result.

Theorem 4.1. Assume that $\left\{\lambda_{n}\right\}$ and $\left\{\mu_{n}\right\}$ are two complex sequences such that $\lambda_{0}=\mu_{0}$ and

$$
\lambda_{n}=\mu_{n}+a \quad \text { and } \quad \lambda_{-n}=\mu_{-n}-b \quad \text { for } \quad n>0,
$$

where $a \geq 0, b \geq 0$ are constants. Suppose $\mu_{n}=\beta_{n}+i \sigma_{n}$ and $\sigma_{n}=\psi(|n|)$, where $\psi(x)$ is increasing and satisfies $\psi(2 x) \leq 2 \psi(x)$ for $x>x_{0}>0$ and $\sum \frac{\psi^{2}(|n|)}{n^{2}}<\infty$.

If $\left|\operatorname{Re} \mu_{n}-n\right| \leq c$ for all integers $n$, then $E_{2}(\lambda) \leq E_{2}(\mu)$ on the interval $(-\pi, \pi)$.

Proof. Suppose $\left\{\mu_{n}\right\}_{-\infty}^{\infty}$ is exact. To prove Theorem 4.1, it suffices to show that $\left\{\lambda_{n}\right\}_{n \in \mathbf{Z} \backslash\{0\}}$ is incomplete. From Proposition A we know that there is an entire function $F_{1}(z)$ vanishing on the sequence $\left\{\mu_{n}\right\}_{n \in \mathbf{Z} \backslash\{0\}}$ such that $F_{1}(x) \in$ $L^{2}(-\infty, \infty)$. From the condition on the sequence $\left\{\lambda_{n}\right\}$, we have $\lambda_{n}=\alpha_{n}+i \sigma_{n}$, where $\alpha_{n}$ and $\sigma_{n}$ are real, and

$$
\alpha_{n}=\beta_{n}+a, \quad \alpha_{-n}=\beta_{-n}-b, \quad n>0 .
$$

It follows from the conditions $\left|\beta_{n}-n\right| \leq c$ and $\sigma_{n}=\psi(|n|)$ that

$$
\begin{aligned}
\sum_{n=1}^{\infty}\left|\frac{1}{\mu_{n}}+\frac{1}{\mu_{-n}}\right| & =\sum_{n=1}^{\infty}\left|\frac{\left(\mu_{n}-n\right)+\left(\mu_{-n}+n\right)}{\mu_{n} \mu_{-n}}\right| \\
& \leq \sum_{n=1}^{\infty}\left(\frac{2 c}{\left|\mu_{n}\right|\left|\mu_{-n}\right|}+\frac{2 \sigma_{n}}{\left|\mu_{n}\right|\left|\mu_{-n}\right|}\right)<\infty .
\end{aligned}
$$

Thus by Hadamard's factorization theorem, we have

$$
F_{1}(z)=K e^{a z}\left(z-\mu_{0}\right) \prod_{n=1}^{\infty}\left(1-\frac{z}{\mu_{-n}}\right)\left(1-\frac{z}{\mu_{n}}\right)
$$

for some constant $a$. As in the proof of Theorem 3.2, we may assume that $\mu_{0}=$ $\lambda_{0}=0$ and

$$
\operatorname{Re} \mu_{n}<0 \text { for } n<0 \text { and } \operatorname{Re} \mu_{n} \geq 0 \text { for } n \geq 0 \text {. }
$$


Following the argument presented in the proof of Theorem 3.2, we set

$$
\begin{aligned}
& G_{1}(z)=K e^{a z} z \prod_{n=1}^{\infty}\left(1-\frac{z}{\beta_{-n}}\right)\left(1-\frac{z}{\beta_{n}}\right), \\
& G_{2}(z)=K e^{a z} z \prod_{n=1}^{\infty}\left(1-\frac{z}{\alpha_{-n}}\right)\left(1-\frac{z}{\alpha_{n}}\right), \\
& F_{2}(z)=K e^{a z} z \prod_{n=1}^{\infty}\left(1-\frac{z}{\lambda_{-n}}\right)\left(1-\frac{z}{\lambda_{n}}\right) .
\end{aligned}
$$

Then from Proposition E, it suffices to show that for some constant $C$

$$
\left|F_{2}(x-i)\right| \leq C\left|F_{1}(x-i)\right|
$$

on the $x$-axis.

From the hypotheses of the theorem, we have that $\sum \frac{\psi^{2}(|n|)}{n^{2}}<\infty$ and $\left|\alpha_{n}-n\right| \leq$ const, so $\sum^{\prime} \frac{\sigma_{n}^{2}}{\alpha_{n}^{2}}$ converges. It follows that

$$
\prod^{\prime}\left|\frac{\alpha_{n}}{\lambda_{n}}\right|=\exp \left\{-\frac{1}{2} \sum^{\prime} \log \left(1+\frac{\sigma_{n}^{2}}{\alpha_{n}^{2}}\right)\right\}
$$

converges to a nonzero constant $A$. Consequently,

$$
\begin{aligned}
\left|\frac{F_{2}(z)}{G_{2}(z)}\right| & =\prod_{n=1}^{\infty}\left|\frac{\left(1-\frac{z}{\lambda_{-n}}\right)\left(1-\frac{z}{\lambda_{n}}\right)}{\left(1-\frac{z}{\alpha_{-n}}\right)\left(1-\frac{z}{\alpha_{n}}\right)}\right|=\prod_{n=1}^{\infty}\left|\frac{\alpha_{-n}}{\lambda_{-n}} \frac{\alpha_{n}}{\lambda_{n}} \frac{\left(z-\lambda_{-n}\right)\left(z-\lambda_{n}\right)}{\left(z-\alpha_{-n}\right)\left(z-\alpha_{n}\right)}\right| \\
& =A\left|\phi_{-}(z)\right|\left|\phi_{+}(z)\right|,
\end{aligned}
$$

where $\phi_{-}(z)=\prod_{n=1}^{\infty} \frac{z-\lambda_{-n}}{z-\alpha_{-n}}$ and $\phi_{+}(z)=\prod_{n=1}^{\infty} \frac{z-\lambda_{n}}{z-\alpha_{n}}$. Note that

$$
\begin{aligned}
\left|\phi_{-}(x-i)\right| & =\prod_{n=1}^{\infty}\left|\frac{\left(x-\alpha_{-n}\right)-\left(\sigma_{-n}+1\right) i}{\left(x-\alpha_{-n}\right)-i}\right|=\prod_{n=1}^{\infty}\left|1+\frac{\sigma_{-n}^{2}+2 \sigma_{-n}}{\left(x-\alpha_{-n}\right)^{2}+1}\right| \\
& =\exp \left\{\frac{1}{2} \sum \log \left|1+\frac{\sigma_{-n}^{2}+2 \sigma_{-n}}{\left(x-\alpha_{-n}\right)^{2}+1}\right|\right\},
\end{aligned}
$$

which converges. Furthermore $\left|\phi_{-}(x-i)\right| \rightarrow 1$ and $\left|\frac{F_{2}(x-i)}{G_{2}(x-i)}\right| /\left|\phi_{+}(x-i)\right| \rightarrow A$ as $x \rightarrow+\infty$. Similarly, we have $\left|\phi_{+}(x-i)\right| \rightarrow 1$ and $\left|\frac{F_{2}(x-i)}{G_{2}(x-i)}\right| /\left|\phi_{-}(x-i)\right| \rightarrow A$ as $x \rightarrow-\infty$.

Next consider $F_{1}(z) / G_{1}(z)$. Similar to the above discussion, we have

$$
\prod_{n=1}^{\infty}\left|\frac{\left(1-\frac{z}{\mu_{-n}}\right)\left(1-\frac{z}{\mu_{n}}\right)}{\left(1-\frac{z}{\beta_{-n}}\right)\left(1-\frac{z}{\beta_{n}}\right)}\right|=B\left|\psi_{-}(z)\right|\left|\psi_{+}(z)\right|,
$$

where

$$
B=\prod_{n=1}^{\infty}\left|\frac{\beta_{-n}}{\mu_{-n}} \frac{\beta_{n}}{\mu_{n}}\right|, \quad \psi_{-}(z)=\prod_{n=1}^{\infty} \frac{z-\mu_{-n}}{z-\beta_{-n}} \quad \text { and } \quad \psi_{+}(z)=\prod_{n=1}^{\infty} \frac{z-\mu_{n}}{z-\beta_{n}} .
$$

Repeating the argument above, we get that

$$
\left|\psi_{-}(x-i)\right| \rightarrow 1 \quad \text { as } \quad x \rightarrow+\infty \text { and }\left|\psi_{+}(x-i)\right| \rightarrow 1 \quad \text { as } \quad x \rightarrow-\infty .
$$


Consequently,

$$
\left|\frac{F_{1}(x-i)}{G_{1}(x-i)}\right| /\left|\psi_{+}(x-i)\right| \rightarrow B \quad \text { as } \quad x \rightarrow+\infty
$$

and

$$
\left|\frac{F_{1}(x-i)}{G_{1}(x-i)}\right| /\left|\psi_{-}(x-i)\right| \rightarrow B \quad \text { as } \quad x \rightarrow-\infty .
$$

Note that when $n>0, \lambda_{n}=\mu_{n}+a$, and when $n<0, \lambda_{n}=\mu_{n}-b$. So we have that

$$
\phi_{+}(x-i)=\prod_{n=1}^{\infty} \frac{x-i-\lambda_{n}}{x-i-\alpha_{n}}=\prod_{n=1}^{\infty} \frac{(x-i-a)-\mu_{n}}{(x-i-a)-\beta_{n}}=\psi_{+}(x-a-i)
$$

and that $\phi_{-}(x-i)=\psi_{-}(x+b-i)$.

To prove (4.1) for all $x \in \mathbf{R}$, we consider the three cases: $x<-M, x>M$ and $-M \leq x \leq M$ for some sufficiently large positive $M$. First for $x<-M$, we have that

$$
\begin{aligned}
\left|F_{2}(x-i)\right| & =\left|\frac{F_{2}(x-i)}{G_{2}(x-i)}\right|\left|G_{2}(x-i)\right| \leq 2 A\left|\phi_{-}(x-i)\right|\left|G_{2}(x-i)\right| \\
& =2 A\left|\psi_{-}(x-i+b)\right|\left|G_{2}(x-i)\right| \\
& \leq \frac{4 A}{B}\left|\frac{F_{1}(x-i+b)}{G_{1}(x-i+b)}\right|\left|G_{2}(x-i)\right| .
\end{aligned}
$$

From Lemma 4.2 below, it is sufficient to show that $\left|\frac{G_{2}(x-i)}{G_{1}(x-i+b)}\right|$ is uniformly bounded. Note that

$$
\begin{aligned}
\frac{G_{2}(x-i)}{G_{1}(x+b-i)} & =\prod_{n=1}^{\infty} \frac{\left(1-\frac{x-i}{\alpha_{-n}}\right)\left(1-\frac{x-i}{\alpha_{n}}\right)}{\left(1-\frac{x-i+b}{\beta_{-n}}\right)\left(1-\frac{x-i+b}{\beta_{n}}\right)}=\prod_{n=1}^{\infty} \frac{\beta_{-n} \beta_{n}}{\alpha_{-n} \alpha_{n}} \frac{x-i-\alpha_{n}}{x-i-\beta_{n}+b} \\
& =\prod_{n=1}^{\infty} L(n, x),
\end{aligned}
$$

where

$$
\begin{aligned}
L(n, x)= & \frac{\left[\alpha_{-n} \alpha_{n}+\left(b \alpha_{n}-a \alpha_{-n}\right)-a b\right]\left[x-i-\alpha_{n}\right]}{\alpha_{-n} \alpha_{n}\left(x-i-\alpha_{n}+a+b\right)} \\
= & \frac{\alpha_{-n} \alpha_{n}\left(x-i-\alpha_{n}\right)+\left(b \alpha_{n}-a \alpha_{-n}-a b\right)\left(x-i-\alpha_{n}\right)}{\alpha_{-n} \alpha_{n}\left(x-i-\alpha_{n}+a+b\right)} \\
= & 1+\frac{\left\{-(a+b) \alpha_{n} \alpha_{-n}+\left(b \alpha_{n}-a \alpha_{-n}-a b\right)\left(-\alpha_{n}\right)\right\}}{\alpha_{-n} \alpha_{n}\left(x-i-\alpha_{n}+a+b\right)} \\
& +\frac{(x-i)\left(b \alpha_{n}-a \alpha_{-n}-a b\right)}{\alpha_{-n} \alpha_{n}\left(x-i-\alpha_{n}+a+b\right)} .
\end{aligned}
$$

Set $\alpha_{n}=n+c_{n}$ for all $n \in \mathbf{Z}$. Then $L(n, x)$ can be written as

$$
\begin{aligned}
L(n, x)= & +\frac{(x-i)\left(n(a+b)+\left(b c_{n}-a c_{-n}-a b\right)\right)}{\alpha_{-n} \alpha_{n}\left(x-i-\alpha_{n}+a+b\right)} \\
& +\frac{n\left(-b c_{-n}-b c_{n}+a b\right)+b\left(c_{n} c_{-n}+c_{n}^{2}+a c_{n}\right)}{\alpha_{-n} \alpha_{n}\left(x-i-\alpha_{n}+a+b\right)} \\
= & 1+\frac{(x-i) K_{n}^{(1)}+K_{n}^{(2)}}{\alpha_{-n}\left(x-i-\alpha_{n}+a+b\right)},
\end{aligned}
$$


where both $K_{n}^{(1)}$ and $K_{n}^{(2)}$ are real and can be bounded with a bound independent of $n$. Moreover, $K_{n}^{(1)} \rightarrow(a+b)>0$ as $n \rightarrow \infty$. Noting that the denominator of the last expression contains a term in $n^{2}$, it follows that for any given $x$, the infinite product $\prod L(n, x)$ converges. Next we show that $L(n, x)$ is uniformly bounded for $x<-M$ :

$$
\begin{aligned}
& L(n, x)=1+\frac{\left[(x-i) K_{n}^{(1)}+K_{n}^{(2)}\right]\left[x-\alpha_{n}+a+b+i\right]}{\alpha_{-n}\left(\left(x-\alpha_{n}+a+b\right)^{2}+1\right)} \\
& =1+\frac{\left[\left(x K_{n}^{(1)}+K_{n}^{(2)}\right)\left(x-\alpha_{n}+a+b\right)+K_{n}^{(1)}\right]+i\left[K_{n}^{(1)}\left(\alpha_{n}-a-b\right)+K_{n}^{(2)}\right]}{\alpha_{-n}\left(\left(x-\alpha_{n}+a+b\right)^{2}+1\right)} \\
& =1+\frac{\left(x K_{n}^{(1)}+K_{n}^{(2)}\right)+\frac{K_{n}^{(1)}}{\left(x-\alpha_{n}+a+b\right)}+i \frac{K_{n}^{(1)}\left(\alpha_{n}-a-b\right)+K_{n}^{(2)}}{\left(x-\alpha_{n}+a+b\right)}}{\alpha_{-n}\left(x-\alpha_{n}+a+b+\delta_{n}\right)} \\
& =1+\frac{\left(x K_{n}^{(1)}+K_{n}^{(3)}\right)-i K_{n}^{(4)}}{\alpha_{-n}\left(x-\alpha_{n}+a+b+\delta_{n}\right)} \\
& =\left\{1+\frac{(-x) K_{n}^{(1)}-K_{n}^{(3)}}{\alpha_{-n}\left(\alpha_{n}-a-b-\delta_{n}-x\right)}\right\}+i \frac{K_{n}^{(4)}}{\alpha_{-n}\left(\alpha_{n}-a-b-\delta_{n}-x\right)},
\end{aligned}
$$

where $\delta_{n}=\frac{1}{x-\alpha_{n}+a+b}$ and $K_{n}^{(3)}$ and $K_{n}^{(4)}$ can be bounded with a bound independent of $n$ and $x$. Let $\omega_{n}=\alpha_{-n}\left(\alpha_{n}-a-b-\delta_{n}-x\right)$ and $\Gamma_{n}=(-x) K_{n}^{(1)}-K_{n}^{(3)}$. Then we have that

$$
\begin{gathered}
1-|L(n, x)|^{2}=-2 \frac{\Gamma_{n}}{\omega_{n}}-\frac{\Gamma_{n}^{2}+\left(K_{n}^{(4)}\right)^{2}}{\left(\omega_{n}\right)^{2}}=\frac{\Gamma_{n}\left[-2 \omega_{n}-\Gamma_{n}\right]-\left(K_{n}^{(4)}\right)^{2}}{\left(\omega_{n}\right)^{2}} \\
=\frac{\Gamma_{n}\left\{(-x)\left(-2 \alpha_{-n}-K_{n}^{(1)}\right)+\left[-2 \omega_{n}-2 \alpha_{-n} x+K_{n}^{(3)}\right]\right\}-\left(K_{n}^{(4)}\right)^{2}}{\left(\omega_{n}\right)^{2}} .
\end{gathered}
$$

Choose $N>0$, independently of $x$, such that when $n>N$, we have $-2 \alpha_{-n}-K_{n}^{(1)}>$ 0 and $-2 \omega_{n}-2 \alpha_{-n} x+K_{n}^{(3)}>1$. Then for sufficiently large $M>0$ and $x<-M$,

$$
1-|L(n, x)|^{2} \geq \frac{\omega_{n}-\left(K_{n}^{(4)}\right)^{2}}{\left(\omega_{n}\right)^{2}}>0 .
$$

It follows that when $x<-M,|L(n, x)|<1$ for $n>N$ and $|L(n, x)|<C(M, N)$ for $n \leq N$. Thus we get that $\left|\frac{G_{2}(x-i)}{G_{1}(x-i+b)}\right|<C_{0}(M, N)$. So combining with Lemma 4.2 below, we have that

$$
\begin{aligned}
\left|F_{2}(x-i)\right| & \leq \frac{4 A C_{0}(M, N)}{B}\left|F_{1}(x-i+b)\right| \\
& \leq \frac{4 A C_{0}(M, N)}{B}|K(x, b)|\left|F_{1}(x-i)\right| \\
& \leq C_{1}(M, N)\left|F_{1}(x-i)\right|
\end{aligned}
$$

for every $x$ satisfying $x<-M$, where $C(M, N), C_{0}(M, N)$ and $C_{1}(M, N)$ are constants. 
Similarly, for $x>M$ we have

$$
\begin{aligned}
L(n, x)= & 1+\frac{(x-i)\left(n(a+b)+\left(b c_{n}-a c_{-n}-a b\right)\right)}{\alpha_{-n} \alpha_{n}\left(x-i-\alpha_{-n}-a-b\right)} \\
& +\frac{n\left(-a c_{-n}-a c_{n}+a b\right)+b\left(c_{n} c_{-n}+c_{-n}^{2}+b c_{-n}\right)}{\alpha_{-n} \alpha_{n}\left(x-i-\alpha_{-n}-a-b\right)} \\
= & 1+\frac{(x-i) K_{1}+K_{2}}{\alpha_{-n}\left(x-i-\alpha_{-n}-a-b\right)},
\end{aligned}
$$

where $K_{1}$ satisfies $0<\frac{a+b}{2}<K_{1}<2(a+b)$ and $K_{2}$ is bounded. Repeating the argument above we can show that $\left|F_{2}(x-i)\right| \leq C_{2}(M, N)\left|F_{1}(x-i)\right|$ for any $x$ satisfying $x>M$.

From Proposition $\mathrm{F}$ below (see Section 5 ), we can assume that $\sigma_{n} \neq-1$ for all $n \in \mathbf{Z}$. Then it is obvious that $\left|F_{2}(x-i)\right| \leq C_{3}(M, N)\left|F_{1}(x-i)\right|$ for any $x$ satisfying $-M \leq x \leq M$. Thus $\left|F_{2}(x-i)\right|$ is uniformly bounded by $\left|F_{1}(x-i)\right|$ for any $x \in \mathbf{R}$. This ends the proof.

Lemma 4.2. Assume that $\left\{\mu_{n}\right\}$ is defined as in Theorem 4.1. Then for any given constant $b, K(x, b)=\prod_{n=-\infty}^{\infty} \frac{\mu_{n}-x+i+b}{\mu_{n}-x+i}$ is bounded unformly on the $x$-axis.

Proof. From the definition of $K(x, b)$, we can rewrite it as

$$
\begin{aligned}
K(x, b) & =\prod_{n=-\infty}^{\infty}\left(1+\frac{b}{\mu_{n}-x+i}\right)=\prod_{m=-\infty}^{\infty}\left(1+\frac{b}{\mu_{m+[x]}-x+i}\right) \\
& =\left(1+\frac{b}{\mu_{[x]}-x+i}\right) \prod_{m=1}^{\infty}\left(1+\frac{b\left(\mu_{m+[x]}+\mu_{-m+[x]}-2 x+2 i\right)+b^{2}}{\left(\mu_{m+[x]}-x+i\right)\left(\mu_{-m+[x]}-x+i\right)}\right) .
\end{aligned}
$$

Since $\mu_{n}=\beta_{n}+i \sigma_{n}$ with $\left|\beta_{n}-n\right|=O(1)$, we have $\mu_{m+[x]}-x=\beta_{m}^{\prime}+i \sigma_{m+[x]}$ with $\beta_{m}^{\prime}=\left(\beta_{m+[x]}-x\right)$. From the restriction above on $\beta_{m}$, we know that $\beta_{m}^{\prime}-m=c_{m}$ and $\left|c_{m}\right| \leq c$ for $m \in \mathbf{Z}$. Thus

$$
\begin{aligned}
K(x, b)= & \left(1+\frac{b}{\mu_{[x]}-x+i}\right) \\
& \times \prod_{m=1}^{\infty}\left(1+\frac{b\left(c_{m}+c_{-m}+b\right)+i b\left(\sigma_{m+[x]}+\sigma_{-m+[x]}+2\right)}{\left(\beta_{m}^{\prime}+i\left(\sigma_{m+[x]}+1\right)\right)\left(\beta_{-m}^{\prime}+i\left(\sigma_{-m+[x]}+1\right)\right)}\right) .
\end{aligned}
$$

Next, we try to prove that for a sufficiently large $x$ with $x>0, K(x, b)$ is bounded uniformly. Choose $x_{0}$ such that when $x>x_{0}, \psi(x)<\frac{x}{2}$ and $\psi(x)<2 \psi\left(\frac{x}{2}\right)$, then

$$
\begin{aligned}
K(x, b) & \leq C \prod_{m=3}^{\infty}\left(1+\frac{\psi(m+[x])+\psi(|[x]-m|)}{\sqrt{m^{2}+\psi^{2}(m+[x])} \sqrt{m^{2}+\psi^{2}(|[x]-m|)}}\right) \\
& =C \prod_{m=3}^{\infty}(1+M(m, x)) .
\end{aligned}
$$

To prove the uniform boundedness of $K(x, b)$, we only need to consider the following summation:

$$
\left(\sum_{m=3}^{[\psi(x)]-1}+\sum_{m=[\psi(x)]}^{[x]}+\sum_{m=[x]+1}^{\infty}\right) M(m, x)=S_{1}+S_{2}+S_{3} .
$$


1) When $3 \leq m<|\psi(x)|$ and $x>x_{0}$, we know that

$$
S_{1} \leq 2 \sum_{m=3}^{[\psi(x)]-1} \frac{1}{\sqrt{m^{2}+\psi^{2}(|[x]-m|)}} \leq 2 \sum_{m=3}^{[\psi(x)]-1} \frac{1}{\psi\left(\frac{x}{2}\right)} \leq 4 .
$$

2) When $\psi(x) \leq m<[x]$, we have $S_{2} \leq 2 \sum_{[\psi(x)]}^{[x]} \frac{\psi(m+x)}{m^{2}}$. Since $\psi(2 x) \leq 2 \psi(x)$ for $x>x_{0}$, we get that

$$
S_{2} \leq 2 \psi(2 x) \sum_{m=[\psi(x)]}^{[x]} \frac{1}{m^{2}} \leq 4 \psi(x)\left(\frac{1}{[\psi(x)]}-\frac{1}{[x]}\right),
$$

which is uniformly bounded.

3) When $m \geq[x]$ and $x>x_{0}$, we have

$$
S_{3} \leq 2 \sum_{m=[x]}^{\infty} \frac{\psi(m+[x])}{m^{2}} \leq \sum_{m=[x]}^{\infty} \frac{\psi(2 m)}{m^{2}},
$$

which is also uniformly bounded.

Thus $\sum_{m=1}^{\infty} M(m, x)$ is uniformly bounded when $x>x_{0}$. For $0<x \leq x_{0}$, it is obvious that the sum of $M(m, x)$ is also bounded uniformly. The same argument can be used to the case of $x<0$. Thus $K(m, x)$ is uniformly bounded on the $\mathrm{x}$-axis.

Remark 4.3. The proof above is based on the evaluation of the sum of $M(m, x)$. Since for a fixed $m, \frac{t}{\sqrt{m^{2}+t^{2}}}$ is increasing with $t$, it follows that

$$
\frac{\psi([x]+m)}{\sqrt{m^{2}+\psi^{2}([x]+m)}} \geq \frac{\psi(x)}{\sqrt{m^{2}+\psi^{2}(x)}} .
$$

If there exists $x_{j} \rightarrow \infty$ such that $\psi\left(\left[x_{j}\right]-i\right)=o\left(\psi\left(\left[x_{j}\right]\right)\right)$ for $i=1, \ldots,\left[x_{j}\right]$, then

$$
\frac{\psi\left(\left[x_{j}\right]+m\right)}{\sqrt{m^{2}+\psi^{2}\left(\left[x_{j}\right]+m\right)} \sqrt{m^{2}+\psi^{2}\left(\left[x_{j}\right]-m\right)}} \geq \frac{\psi\left(\left[x_{j}\right]\right)}{\sqrt{m^{2}+\psi^{2}\left(\left[x_{j}\right]\right)}\left(m+o\left(\psi\left(x_{j}\right)\right)\right)} .
$$

Since $\psi\left(x_{j}\right)=o\left(x_{j}\right)$, a straightforward evaluation yields that

$$
\int_{1}^{x_{j}} \frac{\psi\left(x_{j}\right)}{\sqrt{u^{2}+\psi^{2}\left(x_{j}\right)} u} d u \approx \log \left(\psi\left(x_{j}\right)\right)
$$

which implies that the $\prod\left(1+M\left(m, x_{j}\right)\right)$ may increase with $x_{j}$ for some particular sequences $\left\{\mu_{n}\right\}$.

This explains why we had to impose some restriction on the growth of the imaginary parts of the sequence $\mu_{n}$. It is an open question whether Theorem 4.1 holds without any such restriction. Note that the condition imposed on $\psi(x)$ in Theorem 4.1 is satisfied by many smoothly increasing functions such as $(\log x)^{\alpha}$ or $x^{\alpha}$ $\left(0<\alpha<\frac{1}{2}\right)$.

\section{Completeness of the solution System}

In this section we wish to apply the result of Section 3 to the special sequences introduced in Section 2. In particular we will prove:

Theorem 5.1. Suppose $\left\{V_{n}(a)\right\}$ is defined by (2.4). Then the excess $E_{2}(V)$ of $\left\{e^{i V_{n}(a) t}\right\}$ in $L^{2}(-\pi, \pi)$ satisfies $E_{2}(V)=0$ if $a<0$ and $E_{2}(V)=1$ if $a>0$. 
From (2.4) and Lemma 2.5, we then get:

Corollary 5.2. Let $W_{n}(a)$ be the characteristic roots of the equation $y^{\prime}(t)=$ $a y(t-1)$, where $a \in \mathbf{R}, a \neq 0$. Then the solution system $\left\{e^{W_{n}(a) t}\right\}$ is complete in $L^{2}\left(-\frac{1}{2}, \frac{1}{2}\right)$ and its excess is equal to 1 .

We show in Section 6 that Corollary 5.2 (and thus Theorem 5.1) is best possible in the sense that the solution system $\left\{e^{W_{n}(a) t}\right\}$ fails to be complete on any interval of length greater than one.

It is known that for $\left\{e^{i \lambda_{n} t}\right\}$ to be an unconditional basis, it is necessary that the sequence $\left\{\lambda_{n}\right\}$ satisfies the condition

$$
\inf _{m} \prod_{k \neq m}\left|\frac{\lambda_{k}-\lambda_{m}}{\lambda_{k}-\bar{\lambda}_{m}}\right|>0
$$

See for example 14]. For (5.1) to hold, it is necessary that the sequence satisfies

$$
\left|\frac{\lambda_{k}-\lambda_{n}}{\lambda_{k}-\bar{\lambda}_{n}}\right| \geq a>0, \quad k \neq n .
$$

See [7, Chap VII, Theorem 1.1]. Since clearly $\lambda_{n}=V_{n}(a)$ does not satisfy (5.2), we have:

Proposition 5.3. The system $\left\{e^{W_{n}(a) t}\right\}_{n \in \mathbf{Z} \backslash\{0\}}$ is not an unconditional basis in $L^{2}(-\gamma, \gamma)$, for any $\gamma>0$.

Note that a complete description of unconditional bases in $L^{2}(-\gamma, \gamma)$ of the form $e^{i \lambda_{n} t}$ was given by A.M. Minkin [14. For a direct proof that $\left\{e^{W_{n}(-1 / e) t}\right\}, n \neq 0$, is not even a Schauder basis in $L^{2}\left(-\frac{1}{2}, \frac{1}{2}\right)$, see [29].

Before proving Theorem 5.1 we need to introduce some results. In 1977, Redheffer proved the following.

Proposition F (R.M. Redheffer, [16]). Let $\left\{\lambda_{n}\right\}$ and $\left\{\mu_{n}\right\}$ be two sequences of complex numbers, and suppose that

$$
\operatorname{Re}\left(\lambda_{n}\right)=\operatorname{Re}\left(\mu_{n}\right) \quad \text { and } \quad\left|\operatorname{Im} \lambda_{n}-\operatorname{Im} \mu_{n}\right| \leq \text { const. }
$$

Then the $L^{2}$ excesses of the two exponential systems $\left\{e^{i \lambda_{n} t}\right\}$ and $\left\{e^{i \mu_{n} t}\right\}$ are equal, i.e. $E_{2}(\lambda)=E_{2}(\mu)$.

Sedletskii 19 pointed out that the above theorem fails to be true in $L^{1}(-\pi, \pi)$ or $C(-\pi, \pi)$. Furthermore, in 1985 he constructed in $L^{2}(-\pi, \pi)$ an example which shows that, in general, the boundedness condition on the imaginary parts in the above proposition cannot be removed (see [21]). However he obtained the following two results.

Proposition G (A.M. Sedletskii, [19]). Suppose $\left\{h_{n}\right\}$ is a real sequence, and $E_{2}$ is the excess of the exponential system $\left\{e^{i\left(n+i h_{n}\right) t}\right\}$ in $L^{2}(-\pi, \pi)$.

1) If for some $\alpha \in[0, \infty)$

$$
\left|h_{n}\right| \leq \alpha \log |n| \quad\left(|n| \geq n_{0}\right),
$$

then $E_{2} \leq[\alpha \pi]+1$. If, in addition, $\{\alpha \pi\}<\frac{1}{2}$, then $E_{2} \leq[\alpha \pi]$. Here $[x]$ and $\{x\}$ denote, respectively, the integral and fractional parts of $x$.

2) If moreover

$$
\sum^{\prime} \frac{h_{n}^{2}}{n^{2}}<\infty
$$


and

$$
\left|h_{n}\right| \geq \alpha \log |n| \quad\left(|n| \geq n_{0}, \alpha \in[0, \infty)\right),
$$

then $E_{2} \geq[\alpha \pi]$. If, in addition, $\{\alpha \pi\} \geq 1 / 2$, then $E_{2} \geq[\alpha \pi]+1$.

In particular, if condition (5.3) is satisfied and $h_{n} / \log |n| \rightarrow \infty$ as $n \rightarrow \infty$, then the system $\left\{e^{i\left(n+i h_{n}\right) t}\right\}_{n=-\infty}^{\infty}$ has infinite excess in $L^{2}(-\pi, \pi)$.

Proposition H (A.M. Sedletskii, 21]). Assume that the points $\left\{\lambda_{n}\right\}$ and $\left\{\mu_{n}\right\}$ lie in the curvilinear strip $\{z:|y| \leq \phi(|x|)\}$, where $\phi(x)(x \geq 0)$ is a positive nondecreasing function such that

$$
\sum^{\prime} \phi^{2}(|n|) / n^{2}<\infty \quad \text { and } \quad \operatorname{Re} \lambda_{n}=\operatorname{Re} \mu_{n}, \quad n \in \mathbf{Z} .
$$

If $\left|\operatorname{Im} \lambda_{n}\right| \leq\left|\operatorname{Im} \mu_{n}\right|$ for all $n \in \mathbf{Z}$, then $E_{2}(\lambda) \leq E_{2}(\mu)$.

Theorem 5.4. Suppose $\left\{\lambda_{n}\right\}$ is defined by (3.1), $\mu_{n}=\lambda_{n}+i h_{n}$ with $h_{n}=\frac{1}{2 \pi} \log |n|$, and $h_{0}=0$. Then $E_{2}(\mu)=0$, that is, $\left\{e^{i \mu_{n} t}\right\}$ is complete and exact.

The theorem above can be derived from a result of Sedletskii which he proves using estimates of the Mittag-Leffler function (see Sedletskii's formula below). Here we present a new proof based on the Lambert $W$ functions, and the approach used here will also apply for the proof of Theorem 5.1] First we give one more lemma.

Lemma 5.5. The system $\left\{e^{\left(W_{n}\left(-\frac{1}{e}\right)+1\right) t}, n \in \mathbf{Z} \backslash\{0,-1\}\right\}$ is incomplete in $C\left[-\frac{1}{2}, \frac{1}{2}\right]$ (or in $\left.L^{p}\left(-\frac{1}{2}, \frac{1}{2}\right), 1<p<\infty\right)$.

Proof. To prove this lemma, we need to construct an entire function in the PaleyWiener space which vanishes at every point $z_{n}=\frac{1}{i}\left(W_{n}\left(-\frac{1}{e}\right)+1\right)$.

Set $f(z)=\int_{-A}^{A} e^{i z t} d \omega(t)$, where $\omega(t)=(t+A)^{2}$. Then $f(z)$ is a nontrivial entire function of exponential type. Substituting $t$ by $t-A$, we obtain

$$
\begin{aligned}
f(z) & =\int_{0}^{2 A} e^{i z(t-A)} d t^{2}=2 e^{-i A z} \int_{0}^{2 A} t e^{i z t} d t \\
& =\frac{2 e}{-z^{2}} e^{-i A z}\left\{i(2 A z+i) e^{i(2 A z+i)}+\frac{1}{e}\right\} .
\end{aligned}
$$

Simple computation shows that $f(0) \neq 0$. It follows that the zeroes of $f$ are precisely the nonzero solutions of the equation

$$
i(2 A z+i) e^{i(2 A z+i)}=-\frac{1}{e} .
$$

When $A=\frac{1}{2}$, the solutions of (5.4) are exactly the sequence $\left\{\frac{1}{i}\left(W_{n}\left(-\frac{1}{e}\right)+1\right)\right\}_{-\infty}^{\infty}$. Recalling that $W_{n}\left(-\frac{1}{e}\right)=-1$ for $n=0$ and $n=-1$, we obtain that the zeroes of $f(z)$ are exactly $\left\{-i W_{n}\left(-\frac{1}{e}\right)-i, n \in \mathbf{Z} \backslash\{0,-1\}\right\}$. Thus by Proposition A, $\left\{e^{i\left(-i W_{n}\left(-\frac{1}{e}\right)-i\right) t}, n \in \mathbf{Z} \backslash\{0,-1\}\right\}$ is incomplete in $C\left[-\frac{1}{2}, \frac{1}{2}\right]$ (or in $L^{p}\left(-\frac{1}{2}, \frac{1}{2}\right), 1<$ $p<\infty)$.

Proof of Theorem 5.4. Since from Remark 3.1, $\left\{e^{i \lambda_{n} t}, n \in \mathbf{Z}\right\}$ is complete, by Proposition $\mathrm{H}, E_{2}(\mu) \geq 0$. Thus to prove the theorem, we only need to show that $\left\{e^{i\left(\lambda_{n}+i h_{n}\right) t}, n \in \mathbf{Z} \backslash\{0\}\right\}$ is incomplete.

From Lemma 2.5 and Lemma 5.5, we get that $\left\{e^{\frac{1}{2 \pi}\left(W_{n}\left(-\frac{1}{e}\right)+1\right) t}, k \in \mathbf{Z} \backslash\{0,-1\}\right\}$ is incomplete in $L^{2}(-\pi, \pi)$. Set $V_{n}=\frac{1}{2 \pi i}\left\{W_{n}\left(-\frac{1}{e}\right)+1\right\}, n \geq 0$, and $V_{n}=$ $\frac{1}{2 \pi i}\left\{W_{n-1}\left(-\frac{1}{e}\right)+1\right\}, n<0$. Then $\left\{e^{i V_{n} t}, n \in \mathbf{Z} /\{0\}\right\}$ is incomplete in $L^{2}(-\pi, \pi)$. 
Furthermore if we set $V_{n}=\rho_{n}+i \sigma_{n}$, then $\left\{V_{n}\right\}$ and $\left\{\rho_{n}\right\}$ coincide with $\left\{V_{n}\left(-\frac{1}{e}\right)-\right.$ $\left.\frac{i}{2 \pi}\right\}$ and $\left\{\rho_{n}\left(-\frac{1}{e}\right)\right\}$, respectively, as defined before in Section 2. In addition, by Lemma 2.4

$$
\left|\sigma_{n}-\frac{1}{2 \pi} \log \right| n|| \leq \text { const. }
$$

Thus $\left\{e^{i\left(\rho_{n}+i \frac{1}{2 \pi} \log |n|\right) t}, n \neq 0\right\}$ is incomplete in $L^{2}(-\pi, \pi)$ by Proposition F. Since $\rho_{n}=\rho_{n}\left(-\frac{1}{e}\right)$, and $\lambda_{n}$ is defined by (3.1), then by Lemma 2.4 again, we have

$$
\left|\rho_{n}-\lambda_{n}\right|=O\left(\frac{\log |n|}{|n|}\right) \text {. }
$$

It follows that the two sequences $\left\{\rho_{n}+\frac{i}{2 \pi} \log |n|\right\}$ and $\left\{\lambda_{n}+\frac{i}{2 \pi} \log |n|\right\}$ satisfy the hypotheses of Theorem 3.2. Thus the two exponential systems $\left\{e^{i\left(\rho_{n}+\frac{i}{2 \pi} \log |n|\right) t}\right\}$ and $\left\{e^{i\left(\lambda_{n}+\frac{i}{2 \pi} \log |n|\right) t}\right\}$ have the same excess, which means that $\left\{e^{i\left(\lambda_{n}+\frac{i}{2 \pi} \log |n|\right) t}, n \in\right.$ $\mathbf{Z} \backslash\{0\}\}$ is incomplete. This ends the proof of Theorem 5.4

We note that there is a small misprint in the paper [19] of A.M. Sedletskii from 1978. He actually proved the following formula.

Sedletskii's formula (A.M. Sedletskii, [19]). Suppose $\mu_{n}=n-\beta \operatorname{sign}(n)+$ $i \alpha \log |n|, n \in \mathbf{Z} \backslash\{0\}$, where $\alpha \geq 0$, $\beta$ real. Define $\phi(z)$ by

$$
\phi(z)=z \prod^{\prime}\left(1-\frac{z}{\lambda_{n}}\right) .
$$

Then $|\phi(z)|=O\left(|z|^{\alpha \pi+2 \beta}\right)$ on $\operatorname{Im} z=-1$, and

$$
E_{2}(\mu)=\left\{\begin{array}{lll}
{[\alpha \pi+2 \beta]} & \text { if } & \{\alpha \pi+2 \beta\}<\frac{1}{2}, \\
{[\alpha \pi+2 \beta]+1} & \text { if } & \{\alpha \pi+2 \beta\} \geq \frac{1}{2},
\end{array}\right.
$$

where $[x]$ and $\{x\}$ denote, respectively, the integral and fractional parts of $x$.

The above formula immediately yields the following lemma.

Lemma 5.6. Suppose $\left\{\lambda_{n}\right\}$ is defined by (3.2), $\mu_{n}=\lambda_{n}+i h_{n}$ with $h_{n}=\frac{1}{2 \pi} \log |n|$, and $h_{0}=0$. Then $E_{2}(\mu)=[\alpha \pi+2 \beta]=1$.

Proof of Theorem 5.1. Suppose $a<0$, and $\mu_{n}$ is defined as in Theorem 5.4. Then from Lemma 2.4, we get that $\left|\operatorname{Im} V_{n}(a)-\operatorname{Im} \mu_{n}\right| \leq$ const and $\left|\operatorname{Re} V_{n}(a)-\operatorname{Re} \mu_{n}\right| \leq$ $\epsilon_{n}(a)$, which satisfies the conditions on $\phi(|n|)$ in Theorem 3.2. Since $E_{2}(\mu)=0$, following the approach to prove Theorem [5.4, it is easy to see that $E_{2}\left(V_{n}(a)\right)=0$ for $a<0$.

Using Lemma 5.6. Theorem 3.2 and Proposition F, the same argument will prove that $E_{2}\left(V_{n}(a)\right)=1$ for $a>0$.

\section{RADius of COMPLETENESS}

In this section, using the theorem of A. Beurling and P. Malliavin (Theorem C below), we show that the radius of completeness of the sequence $\left\{V_{n}(a)\right\}$ is $\pi$, and thus that radius of completeness of $\left\{-i W_{n}(a)\right\}$ is one-half.

Recall that to an arbitrary sequence $\Lambda=\left\{\lambda_{n}\right\}$ of complex numbers containing no repetition, we can always associate a number $R \in[0, \infty]$ called the radius of 
completeness characterized by the following property (see [10, Chap. IV]):

The linear span of $\left\{e^{i \lambda_{n} t}\right\}, \lambda_{n} \in \Lambda$, is dense in $L^{2}(-L, L)$ for all $L<R_{\Lambda}$, and is not dense in $L^{2}(-L, L)$ when $L>R_{\Lambda}$.

When $L=R_{\Lambda}$, examples show that $\left\{e^{i \lambda_{n} t}\right\}$ may or may not be complete in $L^{2}(-L, L)$. We claim that for $\Lambda=\left\{V_{n}(a)\right\}, R_{\Lambda}$ is equal to $\pi$. The fact that $\left\{e^{i V_{n}(a) t}\right\}$ is complete in $L^{2}(-\pi, \pi)$ is the content of our Theorem 5.1 above.

Assume that $\Lambda^{\prime}$ is a sequence of (strictly) positive real numbers, which may contain repetition but has no accumulation points other than $\infty$. Let $n_{\Lambda^{\prime}}(t)$ be the number of points of $\Lambda^{\prime}$, counting repetition, in $[0, t]$. Fix $D>0$ and let

$$
\mathcal{O}_{D}\left(\Lambda^{\prime}\right)=\left\{t>0: \frac{n_{\Lambda^{\prime}}(\tau)-n_{\Lambda^{\prime}}(t)}{\tau-t}>D \text { for at least one } \tau>t\right\} .
$$

$\mathcal{O}_{D}\left(\Lambda^{\prime}\right)$ is an open set, and thus can be written as the union of at most countably many open intervals, i.e. $\mathcal{O}_{D}\left(\Lambda^{\prime}\right)=\bigcup_{k \geq 0}\left(a_{k}, b_{k}\right) \subset(0, \infty)$.

Set $\left\|\mathcal{O}_{D}\left(\Lambda^{\prime}\right)\right\|=\infty$ if one of the intervals $\left(a_{k}, b_{k}\right)$ is infinite. Otherwise set

$$
\left\|\mathcal{O}_{D}\left(\Lambda^{\prime}\right)\right\|=\sum_{k \geq 1}\left(\frac{b_{k}-a_{k}}{a_{k}}\right)^{2} .
$$

There exists a number $\widetilde{D}_{\Lambda^{\prime}}>0$ such that $\left\|\mathcal{O}_{D}\left(\Lambda^{\prime}\right)\right\|<\infty$, for all $D>\widetilde{D}_{\Lambda^{\prime}}$ and $\left\|\mathcal{O}_{D}\left(\Lambda^{\prime}\right)\right\|=\infty$ whenever $0<D<\widetilde{D}_{\Lambda^{\prime}}$, unless $\left\|\mathcal{O}_{D}\left(\Lambda^{\prime}\right)\right\|<\infty$ for all $D>0$, in which case $\widetilde{D}_{\Lambda}$ is set to be 0 , or $\widetilde{D}_{\Lambda}=\infty$ for all $D>0$, in which case $\widetilde{D}_{\Lambda^{\prime}}$ is set to be $\infty$. See [10, Chap.II $\S$ C.2]. $\widetilde{D}_{\Lambda^{\prime}}$ is called the effective density of the sequence $\Lambda^{\prime}$.

If the sequence contains one value repeated infinitely many times or has an accumulation point different from $\infty$, we set $\widetilde{D}_{\Lambda}$ to be $\infty$.

For a sequence $\Lambda^{\prime} \subset(-\infty, \infty)$, we set $\Lambda_{+}^{\prime}=\Lambda^{\prime} \cap(0, \infty), \Lambda_{-}^{\prime}=\left(-\Lambda^{\prime}\right) \cap(0, \infty)$, and define $\widetilde{D}_{\Lambda^{\prime}}=\max \left\{\widetilde{D}_{\Lambda_{+}^{\prime}}, \widetilde{D}_{\Lambda_{-}^{\prime}}\right\}$.

Theorem C (A. Beurling and P. Malliavin, 3, 10). For a sequence $\Lambda$ in $\mathbf{C}$ containing no repetition, we have $R_{\Lambda}=\pi \widetilde{D}_{\Lambda^{\prime}}$, where

$$
\Lambda^{\prime}=\left\{\lambda_{n}^{\prime}=\frac{1}{\Re\left(1 / \lambda_{n}\right)} ; \lambda_{n} \in \Lambda, \Re\left(\lambda_{n}\right) \neq 0\right\},
$$

unless $\sum_{\lambda_{n} \neq 0}\left|\Im\left(\frac{1}{\lambda_{n}}\right)\right|=\infty$, in which case $R_{\Lambda}=\infty$.

Proposition 6.1. Fix $a \in R \backslash\{0\}$ and let $\Lambda=\left\{V_{n}(a), n \in \mathbf{Z}\right\}$. Then $R_{\Lambda}=\pi$.

Proof. Note that $\sum_{n}\left|\Im\left(\frac{1}{V_{n}(a)}\right)\right|<\infty$, so by Theorem C, it suffices to prove that $\widetilde{D}_{\Lambda^{\prime}}=1$, where $\Lambda^{\prime}=\left\{\lambda_{n}^{\prime}\right\}_{n \in \mathbf{Z}}$ with $\lambda_{n}^{\prime}=1 / \Re\left\{\frac{1}{V_{n}(a)}\right\}$.

Fix $a<0$ (for $a>0$, the case is similar). We will show that $\widetilde{D}_{\Lambda_{+}^{\prime}}=1$, the proof that $\widetilde{D}_{\Lambda_{-}^{\prime}}=1$ being essentially the same. Note that for $\lambda_{n}^{\prime} \in \Lambda_{+}^{\prime}$, we have by Lemma 2.4

$$
\lambda_{n}^{\prime}=n+\frac{1}{4}-\epsilon_{n}^{(2)}(a)+\frac{\frac{1}{4 \pi^{2}} \log ^{2}|n|+O(1)}{n+\frac{1}{4}-\epsilon_{n}^{(2)}(a)},
$$


where $\epsilon_{n}^{(2)}(a)=O\left(\frac{\log n}{n}\right)$, and thus

$$
\lambda_{n}^{\prime}=n+\frac{1}{4}+\epsilon_{n}^{\prime}(a)
$$

with $\epsilon_{n}^{\prime}(a) \rightarrow 0$ as $n \rightarrow \infty$.

We first show that $\widetilde{D}_{\Lambda_{+}^{\prime}} \geq 1$. Fix $0<D<1$. Choose a (large) positive integer $N_{1}$ such that $\frac{1}{N_{1}}<1-D$ and $\lambda_{n}^{\prime} \in\left(n, n+\frac{1}{2}\right)$ if $n \geq N_{1}$. If $n>0$ and $t \in\left[\lambda_{n}^{\prime}, \lambda_{n+1}^{\prime}\right)$, then

$$
\frac{n_{\Lambda_{+}^{\prime}}\left(t+N_{1}\right)-n_{\Lambda_{+}^{\prime}}(t)}{\left(t+N_{1}\right)-t} \geq \frac{n+\left(N_{1}-1\right)-n}{N_{1}}=1-\frac{1}{N_{1}}>D
$$

Thus $\mathcal{O}_{D}\left(\Lambda_{+}^{\prime}\right)=(0, \infty)$ and $\left\|\mathcal{O}_{D}\left(\Lambda^{\prime}\right)\right\|=\infty$ whenever $0<D<1$. This implies $\widetilde{D}_{\Lambda_{+}^{\prime}} \geq 1$.

To prove that $\widetilde{D}_{\Lambda_{+}^{\prime}} \leq 1$, and thus that $\widetilde{D}_{\Lambda^{\prime}+}=1$, we must show that $\left\|\mathcal{O}_{D}\left(\Lambda_{+}^{\prime}\right)\right\|<$ $\infty$ for any $D>1$. For this we only need to establish the existence of a positive integer $N_{2}$ such that $\lambda_{n}^{\prime} \notin \mathcal{O}_{D}\left(\Lambda_{+}^{\prime}\right)$ whenever $n>N_{2}$, since then a straightforward estimate using the asymptotic behaviour of the sequence $\Lambda_{+}^{\prime}$ shows $\left\|\mathcal{O}_{D}\left(\Lambda_{+}^{\prime}\right)\right\|:=$ $\sum\left(b_{k}-a_{k} / a_{k}\right)^{2}$ to be finite.

Fix $D>1$ and $n \geq 1$. If $m \geq 0(m \in \mathbf{Z})$ and $\tau \in\left[\lambda_{n+m}^{\prime}, \lambda_{n+m+1}^{\prime}\right)$, then

$$
\frac{n_{\Lambda_{+}^{\prime}}(\tau)-n_{\Lambda_{+}^{\prime}}\left(\lambda_{n}^{\prime}\right)}{\tau-\lambda_{n}^{\prime}}<\frac{m}{\lambda_{n+m}^{\prime}-\lambda_{n}^{\prime}}=\frac{m}{m+\left(\epsilon_{n+m}^{\prime}(a)-\epsilon_{n}^{\prime}(a)\right)} .
$$

Since $\epsilon_{n}^{\prime}(a) \rightarrow 0$ as $n \rightarrow \infty$, there exists an integer $N_{2}$ such that for all $n \geq N_{2}$ and all $m \geq 0, \frac{m}{m+\left(\epsilon_{n+m}^{\prime}(a)-\epsilon_{n}^{\prime}(a)\right)}<D$, and thus the inequality $\frac{n_{\Lambda_{+}^{\prime}}(\tau)-n_{\Lambda_{+}^{\prime}}\left(\lambda_{n}^{\prime}\right)}{\tau-\lambda_{n}^{\prime}}>D$ holds for no $\tau>\lambda_{n}$ when $n \geq N_{2}$. This implies $\lambda_{n}$ is not in $\mathcal{O}_{D}\left(\Lambda_{+}^{\prime}\right), n \geq N_{2}$, and ends the proof.

\section{ACKNOWLEDGEMENTS}

We would like to express our sincere gratitute to Professor R. Corless for bringing the problem of representation of the solutions of delay-differential equations to our attention and for many useful discussions, and to Professor A.M. Sedletskii and an unknown referee for their valuable comments. The second author also would like to take this opportunity to thank Professor Corless for his enthusiastic guidance and encouragement during the time of his graduate study.

\section{REFERENCES}

[1] S. A. Avdonin and I. Joó, Riesz basis of exponentials and sine-type functions, Acta Math. Hungar. 51(1) (1988), 3-14. MR.0934576 (90g:42058)

[2] R. Bellman and K. L. Cooke, Differential-Difference Equations, Academic Press, 1963. MR0147745 (26:5259)

[3] A. Beurling and P. Malliavin, On the closure of characters and the zeros of entire functions, Acta Math. 118 (1967), 79-93. MR0209758 (35:654)

[4] R. P. Boas, Jr., Entire Functions, Academic Press, 1954. MR0068627 (16:914f)

[5] R. M. Corless, G. H. Gonnet, D. E. G. Hare, D. J. Jeffrey and D. E. Knuth, On the Lambert $W$ function, Advances in Comp. Math. 5 (1996), 329-359. MR1414285 (98j:33015)

[6] N. Fujii, A. Nakamura, and R. Redheffer, On the excess of sets of complex exponentials, Proc. Amer. Math. Soc. 127 (1999), 1815-1818. MR1476126 (99i:30006)

[7] J. Garnett, Bounded Analytic Functions, Academic Press, 1981. MR0628971 (83g:30037)

[8] S. Jaffard and R. M. Young, A representation theorem for Schauder bases in Hilbert space, Proc. Amer. Math. Soc. 126 (2) (1998), 553-560. MR1425127(98d:46019) 
[9] M. I. Kadec', The exact value of the Paley-Wiener constant (Russian), Dokl. Akad. Nauk SSSR 155 (1964), 1253-1254. English translation: Sov. Math. Dokl. 5 (1964), 559-561. MR0162088 (28:5289)

[10] P. Koosis, Lecons sur le Théorème de Beurling et Malliavin, les Publications CRM, (Montréal), 1996. MR1430571 (99e:42023)

[11] B. Ja. Levin, Distribution of zeros of entire functions, Translations of Mathematical Monographs, Vol. 5, Amer. Math. Soc., 1980. MR0589888 (81k:30011)

[12] N. Levinson, On the closure of $\left\{e^{i \lambda_{n} x}\right\}$ and integral functions, Proc. Cambridge Phil. Soc. 31 (1935), 335-346.

[13] N. Levinson, Gap and Density Theorems, AMS. Col. Public., Vol. 26, 1940. MR0003208 $(2: 180 \mathrm{~d})$

[14] A. M. Minkin, The reflection of indices and unconditional bases of exponentials (Russian), Algebra i Analiz 3 (1991), 109-134. English translation: St. Petersburg Math. J. 3 (1992), 1043-1068. MR 1186238 (95a:42051)

[15] R. E. Paley and N. Wiener, Fourier transforms in the complex domain, AMS. Col. Public., Vol. 19, 1934. MR.1451142 (98a:01023)

[16] R. M. Redheffer, Completeness of Sets of Complex Exponentials, Advances in Mathematics 24 (1977), 1-62. MR0447542 (56:5852)

[17] R. M. Redheffer and R. M. Young, Completeness and basis properties of complex exponentials, Trans. Amer. Math. Soc. 277 (1983), 93-111. MR0690042 (84c:42047)

[18] A. M. Sedleckii, Excesses of systems of exponential functions (Russian), Mat. Zametki 22 (1977), 803-814. English translation: Math. Notes 22 (1977), 941-947. MR0477593 $(57: 17111)$

[19] A. M. Sedleckii, On completeness of the system $\left\{\exp \left(i x\left(n+i h_{n}\right)\right)\right\}$, Anal. Math. 4 (1978), 125-143. MR0505535 (80g:42021)

[20] A. M. Sedletskii, Excesses of systems, close to one another, of exponentials in $L^{p}$ (Russian), Sibirs. Mat. Zh. 24 (1983), 164-175. English translation: Siberian Math. J. 24 (1983), 626635. MR0713593 (84i:42042)

[21] A. M. Sedletskii, Purely imaginary perturbations of the exponents $\lambda_{n}$ in the system $\left\{\exp \left(i \lambda_{n} t\right)\right\}$ (Russian), Sibirs. Mat. Zh. 26 (1985), 151-158. English translation: Siberian Math. J. 26 (1985), 597-603. MR0804027 (87a:42039)

[22] A. M. Sedletskii, Completeness and nonminimality of systems of exponentials in $L^{p}(-\pi, \pi)$, Sibirs. Mat. Zh. 29 (1988), 159-170. English translation: Siberian Math. J. 29 (1988), 123133. MR0936794 (89j:42010)

[23] A.M. Sedletskii, Fourier transforms and approximations. Gordon and Breach Science Publ., Amsterdam, 2000. MR 1935577 (2003i:42002)

[24] S. Verblunsky, On a class of Cauchy exponential series, Rend. Circ. Mat. Palermo 10 (1961), 5-26. MR0145084 (26:2619)

[25] E. M. Wright, Solution of the equation $z e^{z}=a$, Bull. Amer. Math. Soc. 65 (1959), 89-93. MR 0129130 (23:B2167)

[26] R. M. Young, An Introduction to Nonharmonic Fourier Series, Academic Press, 1980 and 2001. MR0591684(81m:42027) MR1836633(2002b:42001)

[27] R. M. Young, On a theorem of Ingham on nonharmonic Fourier series, Proc. Amer. Math. Soc. 92 (1984), 549-553. MR0760944 (85m:42028)

[28] R. M. Young, On the stability of exponential bases in $L^{2}(-\pi, \pi)$, Proc. Amer. Math. Soc. 100 (1987), 117-122. MR0883412 (88m:42056)

[29] H. Zhong, Non-harmonic Fourier series and applications, Ph.D. thesis, Univ. of Western Ontario, 2000.

Department of Mathematics, University of Western Ontario, London, Ontario, CANADA N6A 5B7

E-mail address: boivin@uwo.ca

Robarts Research Institute, 100 Perth Drive, P.O. Box 5015, London, Ontario, CANADA N6A $5 \mathrm{~K} 8$

Current address: Department of Radiation Oncology, Virginia Commonwealth University, 401 College Street, Richmond, Virginia 23298

E-mail address: hzhong@vcu.edu 